\title{
Inundações no Meio Urbano e Vulnerabilidade Social: estudo de caso em Curitiba, Brasil
}

\author{
Edilberto Nunes Moura \\ Pontifícia Universidade Católica do Paraná - Curitiba - Paraná - Brasil \\ Ariadne Stradiotto Frenzel \\ Pontifícia Universidade Católica do Paraná - Curitiba - Paraná - Brasil \\ Isabela Ignácio de Moura \\ Universidade Tecnológica Federal do Paraná- Curitiba - Paraná - Brasil
}

\section{Resumo}

Este trabalho aborda as relações espaciais da vulnerabilidade social e as áreas sujeitas à inundações na porção central da Bacia Hidrográfica do Rio Belém, em Curitiba/Brasil, tendo como problema de pesquisa o possível agregado dos aspectos de vulnerabilidade ambiental à inundações nos ambientes urbanos em condições sociais previamente existentes. $O$ objetivo do artigo foi identificar regiões de sobreposição da vulnerabilidade social às áreas mais propensas às inundações, obtidas a partir da criação de cenários desse tipo de evento simulados em ambiente SIG, no panorama de vulnerabilidade social identificado pelo Atlas de Vulnerabilidade Social dos Municípios Brasileiros (IPEA, 2015). Os cenários de inundação foram criados através de modelagem computacional com o uso do software Hazus, adaptado às condições brasileiras. A partir daí foi possível identificar possíveis áreas e população afetadas pela massa hídrica simulada. Com os resultados obtidos se pode estimar a população afetada, classificar o grau de vulnerabilidade por inundações e associar à vulnerabilidade social, e assim obter um índice de vulnerabilidade socioambiental devido aos eventos de inundações urbanas.

Palavras-chave: Inundações urbanas. Vulnerabilidade social. Hazus. SIG.

Floods in the Urban Environment and Social Vulnerability: a study case in Curitiba, Brazil

\begin{abstract}
This paper deals with the spatial relationship of social vulnerability and the flooding areas in the central portion of the Belem River Basin, in Curitiba / Brazil, as the research problem being the possible aggregation of aspects of environmental vulnerability to flooding in urban areas under social conditions previously existing. The objective of this article was to identify regions of overlapping social vulnerability to the flooding areas, obtained from the creation of scenarios of this type of event simulated in a GIS environment, in the panorama of social vulnerability identified by the Atlas of Social Vulnerability of Brazilian Municipalities (IPEA, 2015). The flood scenarios were created through computational modeling using the Hazus software, adapted to the Brazilian conditions. Then it was possible to identify possible areas and population affected by the simulated water mass. With the results obtained, it is possible
\end{abstract}


to estimate the affected population, to classify the degree of vulnerability due to floods and to associate it with social vulnerability, and thus to obtain an index of social and environmental vulnerability due to the events of urban floods.

Keywords: Urban Floods. Social Vulnerability. Hazus. GIS.

\section{Inundaciones en Medio Urbano y Vulnerabilidad Social: estudio de caso en Curitiba, Brasil}

\section{Resumen}

Este trabajo aborda las relaciones espaciales de la vulnerabilidad social y las áreas sujetas a inundaciones en la porción central de la Cuenca Hidrográfica del Río Belém, en Curitiba / Brasil, teniendo como problema de investigación el possible agregado de aspectos de vulnerabilidad ambiental a las inundaciones em los ambientes urbanos en condiciones sociales previamente existentes. El objetivo del artículo fue identificar regiones de superposición de la vulnerabilidad social a las áreas más propensas a las inundaciones, obtenidas a partir de la creación de escenarios de ese tipo de evento simulados en ambiente SIG, en el panorama de vulnerabilidad social identificado por el Atlas de Vulnerabilidad Social de los Municipios Brasilizemos (IPEA, 2015). Los escenarios de inundación fueron creados a través de modelado computacional con el uso del software Hazus, adaptado a las condiciones brasileñas. A partir de ahí fue posible identificar posibles áreas y población afectadas por la masa hídrica simulada. Con los resultados obtenidos se puede estimar la población afectada, clasificar el grado de vulnerabilidad por inundaciones y asociar a la vulnerabilidad social, y así obtener un índice de vulnerabilidad socioambiental debido a los eventos de inundaciones urbanas.

Palabras clave: Inundaciones urbanas. Vulnerabilidad social. Hazus. SIG.

\section{Introdução}

O fenômeno de inundações pode ocorrer devido ao processo hidrológico natural ou às alterações antrópicas vinculadas ao processo de urbanização, como a impermeabilização de grandes porções do território, bem como modificações de cursos d'água, que impedem a absorção do volume da massa de água proveniente de chuvas, impactando o processo de escoamento, gerando sobrecarga na drenagem natural e na drenagem antrópica (TINGSANCHALI, 2012).

O processo de ocupação das cidades brasileiras desencadeou um movimento de fixação populacional em locais impróprios para ocupação, como planícies de inundação, morros, locais com solo frágil, onde diversas situações de risco e vulnerabilidade social e ambiental podem ser observadas, como demonstram estudos no âmbito jurídico - enfocando o direito de acesso à terra urbana, no âmbito da saúde - em análise das condições de saneamento e acesso ao sistema de saúde, no âmbito urbano - destacando conjunturas de vulnerabilidade (MARICATO, 2003). Já na década de 1970, observa-se um aumento da população brasileira como um todo, mas especialmente no incremento da população urbana, com aproximadamente 21 milhões de pessoas a mais habitando centralidades urbanas, em um período de 10 anos, comparado ao Censo Demográfico anterior (IBGE, 2010).

Tucci (2008) aponta que, a partir dos anos 1980, as extensões das periferias urbanas brasileiras cresceram mais do que os núcleos ou municípios centrais das metrópoles, caracterizando alta densidade de ocupação da terra, expansão horizontal das cidades, pobreza e exclusão social. A dinâmica de expansão horizontal nas periferias e intensificação da verticalização aliada com adensamento 
populacional nos centros agravou os problemas estruturais na relação entre os meios natural e antrópico.

No Brasil, a ocupação urbana demonstra exercer grande pressão no sistema natural, com grandes parcelas da sociedade em localizações precárias, com pouca ou inexistente infraestrutura, com o agravante da mesma população não contar com representatividade no contexto urbano, acentuando desvantagens sociais, elevando a vulnerabilidade destes locais e das pessoas que os habitam (HUMMEL et al, 2016).

Neste contexto, o foco da presente pesquisa volta-se para a integração das características de vulnerabilidades social e ambiental da população residente na região central da Bacia Hidrográfica do Rio Belém (Curitiba/Brasil), a partir simulação de massa hídrica oriunda de inundação urbana, criada a partir de cenários desenvolvidos com a técnica de modelagem computacional em ambiente SIG (Sistema de Informação Geográfica).

\subsection{Inundações nos ambientes urbanos brasileiros e seus respectivos impactos}

O processo de ocupação e distribuição da população no ambiente urbano está intimamente relacionado com as dinâmicas econômicas. Rolnik e Klink (2011), em análise da terra urbana, argumentam que "o mercado de terras determina a forma de estruturação da cidade, em conjunto com a política de investimentos urbanos, (...) de infraestrutura e com a relação que ele estabelece com as estratégias de regulação urbana". Dessa forma, a produção do espaço é uma produção de localizações, pois diferentes localizações possuem diferentes valores, que se associam ao valor dos elementos urbanos ali presentes e a maior acessibilidade aos bens e serviços existentes na cidade.

Compreende-se que a maior oferta de infraestrutura e acessos atrai determinada parcela da população, que possui recursos para investir e se manter em locais com maior oferta de serviços, enquanto a parcela restante tende a se localizar em regiões menos desenvolvidas, periféricas e desvalorizadas perante o mercado imobiliário e, em muitos casos, locais potencialmente de risco ambiental (ROLNIK \& KLINK, 2011).

Em se tratando de inundações em ambientes urbanos, as perdas e danos podem ser classificadas como tangíveis ou intangíveis em um primeiro nível, e diretos e indiretos em um segundo nível. Segundo Machado et al. (2005) os danos intangíveis são aqueles ligados diretamente à pessoa como a perda de vidas, e também o aspecto psicológico, visto que eventos de desastres tendem a desencadear transtornos psicológicos em maior ou menor grau, bem como danos à saúde física, devido a exposição da população a doenças de veiculação hídrica, provenientes da sobrecarga no sistema de drenagem.

A mensuração de danos tangíveis, tanto diretos quanto indiretos, podem ser tratados a partir de análises técnicas, a fim de identificar o valor dos mesmos, seus custos, prejuízos devido a interrupção de funcionamento (JONOV et al., 2013). Enquanto os danos intangíveis se tornam de difícil mensuração, devido a multiplicidade de afetados por eventos de inundações e o não-acompanhamento da população atingida nas ações pós-evento.

Considerando o patrimônio físico, estimam-se perdas e danos de edificações de diversas formas, visto que os materiais utilizados nas construções se comportam 
de maneiras distintas quando em contato direto com a massa hídrica, com a facilitação do desenvolvimento de mofo, fungos, bem como a deterioração dos materiais, podendo existir odores desagradáveis. Os danos físicos às construções são frequentemente estimados por meio de orçamentos de reforma, sendo os prejuízos avaliados através dos preços de mercado atualizados (MACHADO et al., 2005).

Um outro transtorno que pode ser causado por eventos de inundação é a ruptura ou perturbação de algum dos serviços essenciais no meio urbano Equipamentos públicos e serviços e Infraestrutura - pode resultar em alteração ou danificação de outros serviços e setores de atividade nas cidades, implicando em danos indiretos e, em alguns casos, em danos diretos. Do mesmo modo, deve-se estimar redução de atividade econômica nos locais atingidos por inundações, o que pode desestabilizar ou incrementar o sistema econômico de determinadas localidades, alterando dinâmicas urbanas (JONOV et al., 2013).

\subsection{Simulações computacionais de manchas de inundação no meio urbano}

Para tentar entender a realidade, muitas vezes são necessárias idealizações e simplicações, que permitem construir objetos-modelo ou modelos conceituais do fenômeno de interesse da situação em pauta, como descreve Bunge (2013). Os modelos conceituais podem dar ao seu construtor uma imagem simbólica do real, com atribuição de certas propriedades ao mesmo, buscando sua inserção em uma teoria capaz de descrevê-lo teórica e matematicamente. Esta é a etapa do modelo teórico, cuja aceitação é provisória, a refutação pode ser definitiva e a revisão (ou modificações) dependem da experimentação. Segundo Veit e Araújo (2010) a modelagem computacional é um dos pilares fundamentais do desenvolvimento científico contemporâneo. Para os autores, o termo modelagem computacional, em geral, está associado com a computação voltada para elaboração de representações computacionais de problemas complexos oriundos das mais diversas áreas do conhecimento.

Tucci (2008) enfoca que o uso da cartografia na identificação e diagnóstico de áreas e populações vulneráveis e de risco tem sido amplamente utilizada em diversas cidades brasileiras e tem em comum, de modo geral, uma combinação de dados referentes a aspectos geológicos, geomorfológicos e de uso do solo, bem como sociodemográficos. $\mathrm{O}$ autor caracteriza o mapeamento de riscos e vulnerabilidades como medida não-estrutural para controle de inundações e demais desastres, enfatizando a regulamentação do uso da terra em áreas inundáveis, definindo a ocupação destas áreas a partir das diferentes cotas de inundação, por exemplo, propondo uso habitacional em cotas com menos riscos, mas com precauções especiais, enquanto para cotas com mais riscos o uso pode ser direcionado para recreação e lazer, com baixo custo de investimento.

Além do mapeamento das características físicas e de ocupação de população vulnerável em eventual local de risco, o diagnóstico e mapeamento social de determinadas áreas é necessário, a fim de mensurar os impactos de um desastre natural - tanto no âmbito de perdas e prejuízos físicos, quanto na causalidade de danos psicológicos e sociais na população afetada (MACHADO et al., 2005).

\section{0 modelo Hazus}


O software Hazus modela e exibe gráficos, imagens e relatórios relativos à ameaças e vulnerabilidades para situações de desastre natural. Essa ferramenta foi desenvolvida pela Federal Emergency Manegement Agency (FEMA), responsável pela gestão de desastres nos Estados Unidos da América. Se trata de um sistema computacional gratuito, que utiliza a plataforma tecnológica de um Sistema de Informação Geográfica (SIG), especificamente o software ArcGIS da ESRI ${ }^{\circledR}$ (SCHNEIDER, 2006). O programa foi projetado para ser utilizado apenas no âmbito dos Estados Unidos da América, porém é passível de utilização em outras regiões, desde que sejam realizadas as devidas adaptações e inserções de dados básicos da região de estudo (KAVECRIS, 2013).

De uma maneira geral, o software avalia riscos de desastres em uma área delimitada e suas eventuais consequências. O desenvolvimento do programa se deu no âmbito de estudo da prevenção e análise de danos provenientes de abalos sísmicos, desastre com grande ocorrência no território da América do Norte, mas obteve repercussão positiva e foram desenvolvidas versões que proporcionam análise multirrisco de desastres como furacões, terremotos e inundações (SCAWTHORN, 2006; SCHNEIDER, 2006). E mais recentemente, análises de tsunamis.

Após a inserção das informações relativas ao local de estudo, o Hazus disponibiliza mapas de avaliação de vulnerabilidade, bem como estima a exposição de determinada população e estruturas construídas a possíveis danos, tendo como objetivo simular cenários para contribuir na gestão e decisões tomadas em eventual desastre (SCAWTHORN, 2006).

Segundo considerações de Schneider (2006), os resultados das análises proporcionadas pelo Hazus, promovem sugestões de remoção e elevação de estruturas de um local sujeito à inundação. Além de sugestões sobre áreas prioritárias, pontos para desobstrução, revitalização de serviços essenciais (abastecimento de água, coleta de esgoto, entre outros), e remarcação do uso e ocupação da terra.

Ainda que os benefícios da implantação do programa sejam de grande impacto, como o deslocamento de populações de áreas de risco e proteção da infraestrutura existente, é necessário que a equipe designada para desenvolvimento, adaptação e operação do software esteja qualificada e alinhada com as especificidades requeridas, a fim de gerar cenários mais próximos da realidade de um possível evento de inundação (HANSEN, 2014). Além destas considerações, se torna necessário averiguar a existência, extensão e confiabilidade dos dados de entrada do programa, de forma que quanto maior a vastidão e credibilidade desses dados, mais alinhados com a realidade do local de estudo serão os resultados, auxiliando na tomada de decisões pelos tomadores de decisão (DING, 2008).

O objetivo perseguido nesse trabalho foi identificar regiões de sobreposição da vulnerabilidade social às áreas mais propensas às inundações na Bacia do Rio Belém em Curitiba, Paraná.

\section{Procedimento Metodológico}

\section{1 Área de estudo}


Para o estudo de caso desenvolvido utilizou-se o recorte geográfico da região central da Bacia Hidrográfica do Rio Belém, inserida em sua totalidade no município de Curitiba/Brasil.

O Rio Belém conta com sua nascente na porção norte do município de Curitiba e segue seu curso pela área central da cidade, toma sentido sudeste e tem sua foz na porção sul do município, desaguando no Rio Iguaçu, tendo 21 km de extensão e 87 $\mathrm{km}^{2}$ de área de bacia, com sua área de drenagem representando aproximadamente $20 \%$ da área do município, abrigando aproximadamente $50 \%$ da população do município (IPARDES, 2012).

O desenvolvimento da ocupação do município de Curitiba se deu na porção central da Bacia Hidrográfica do Rio Belém (BOLLMANN, 2008), em destaque na Figura 1. Desta forma, esta região do município concentra diversos pontos de interesse, não apenas na escala municipal, como na escala regional, englobando a região que concentra as sedes dos poderes Municipal e Estadual, no bairro Centro Cívico, e diversos terminais de transporte coletivo que conectam o município polo da Região Metropolitana aos seus demais municípios. A região central da Bacia Hidrográfica do Rio Belém abriga equipamentos públicos de diversas categorias, especialmente Saúde, Educação, Cultura, Esporte e Infraestrutura, além de exemplares relevantes de projetos de qualidade projetual de arquitetura, afirmando a representatividade e a urbanidade do município de Curitiba (CMC, 2015). 
Figura 1. Localização da área de estudo

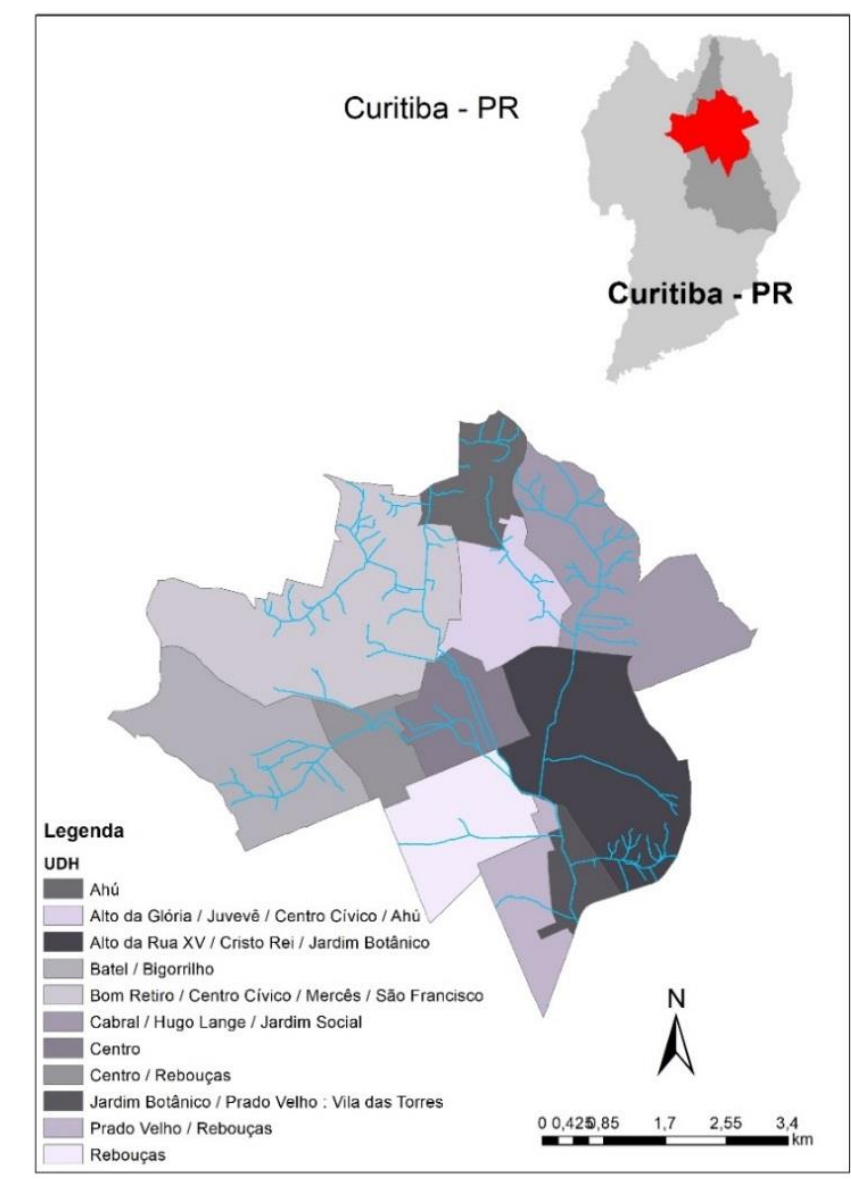

Fonte: IPEA, 2015. IPPUC, 1997; 2012. Modificado pelos autores.

Nesse estudo, foi considerada apenas a porção central da Bacia Hidrográfica do Rio Belém para desenvolvimento dos cenários de inundação. A localidade teve sua escolha pautada a partir de uma condição média da topografia (declividade) e do fluxo d'água dos rios, evitando locais com condições extremas de relevo e àqueles muito próximos da nascente e da foz do rio, onde o comportamento hidráulico recebe influência de outras variáveis.

Nesta unidade de análise, simularam-se potenciais manchas de inundação, criadas a partir de dados digitais da topografia e dados da vazão dos rios para um tempo de retorno de 25 anos.

Tabela 1. Vazão $\left(\mathrm{m}^{3} / \mathrm{s}\right)$ dos rios e tempo de retorno

\begin{tabular}{lcc}
\hline \multicolumn{1}{c}{ Rio } & Tempo de retorno & Vazões $\left(\mathbf{m}^{\mathbf{3}} / \mathbf{s}\right)$ para TR=25 anos \\
\hline Belém & 367,91 \\
Água Verde & 74,58 \\
Juvevê & 25 anos & 76,01 \\
Ivo & 61,00 \\
Pilarzinho & 21,74 \\
Juvevê do Norte & 24,58 \\
\hline
\end{tabular}

Fonte: SUDERHSA, 2002. Plano Diretor de Drenagem da Bacia Hidrográfica do Alto Iguaçu. 


\subsection{0 Índice IVS}

Para este estudo, foram utilizados dados do Índice de Vulnerabilidade Social (IVS) para cada Unidade de Desenvolvimento Humano (UDH) de Curitiba. Os dados são de domínio público, disponibilizados pela plataforma do IPEA, através do Atlas de Vulnerabilidade Social (2015). O IVS é um índice sintético que reúne dezesseis indicadores, estruturados em três dimensões: Infraestrutura Urbana, Capital Humano e Renda e Trabalho, considerando a escala de municípios brasileiros, com base na malha municipal do Censo Demográfico 2010, e para as Unidades de Desenvolvimento Humano (UDH) 1 das principais Regiões Metropolitanas do país.

\subsection{Estrutura e tratamento de dados}

Na criação da extensão e da profundidade das manchas de inundação, foi utilizado um dado do relevo no formato de modelo digital de elevação (DEM - Digital Elevation Model), obtido a partir de processamento de dados laser scanner (LIDAR) do território com resolução espacial de 5 metros, obtidos junto à COPEL (Companhia Paranaense de Energia, 2012).

Os dados demográficos foram extraídos do Censo 2010 do IBGE de forma a obter o número de habitantes por setores censitários inseridos no recorte geográfico.

Para realizar a integração entre os dados do meio físico e os dados demográficos foi utilizado o SIG proprietário ArcGIS@ versão 10.5.1, licença nível Advanced. Essa ferramenta de SIG permitiu a utilização de um operador lógico (booleano), para realizar os cruzamentos entre as camadas de dados geográficos. $\mathrm{E}$ assim foram feitas as operações lógicas do tipo $A$ and $B$ retornando todos os elementos contidos na intersecção entre A e B.

O apelo da abordagem booleana é a sua simplicidade e fácil aplicação, com a combinação lógica de mapas em um SIG diretamente análoga ao tradicional método de sobreposição empregado nas mesas de luz. Apesar de sabermos que, na prática, pode não ser apropriado atribuir igual importância para cada um dos critérios combinados (CÂMARA, 1995).

O resultado desse procedimento foi a projeção da população presente em cada nível de profundidade.

Em cada intervalo de profundidade foram atribuídos valores de ponderação em um intervalo de 0 a 1, considerando que quanto maior a profundidade atingida pela mancha de inundação simulada, maior o possível impacto à população e assim mais próximo de 1 será o valor atribuído.

Cada nível de profundidade foi multiplicado ao percentual da população inserida no respectivo nível, resultando em uma nota obtida em cada situação. E

\footnotetext{
${ }^{1}$ As UDH foram delineadas buscando áreas mais homogêneas das condições socioeconômicas, do que as áreas de ponderação do IBGE. As UDHs são construídas com o objetivo de melhor captar a diversidade de situações relacionadas com o desenvolvimento humano que ocorre no interior dos espaços intrametropolitanos. As UDHs estão voltadas para a análise espacial das Regiões Metropolitanas (RM) por meio de recortes espaciais com o objetivo de retratar as desigualdades de forma mais contundente. Cf. ATLAS BRASIL 2013. Atlas da vulnerabilidade social nos municípios brasileiros. Brasília, 2015.
} 
assim, obter o nível de impacto final em cada UDH, representado pelo somatório das notas obtidas em cada profundidade atingida.

Na obtenção do índice de vulnerabilidade devido à inundação para cada UDH do recorte geográfico, foram consideradas as profundidades de inundação atingidas em cada UDH, suas respectivas áreas de abrangência e a população inserida em cada profundidade.

\section{Resultados}

A Tabela 2 mostra as áreas atingidas pela mancha de inundação e a proporção de suas profundidades para as UDH envolvidas no estudo.

Podemos observar que as UDH Centro e Centro/Rebouças são aquelas com o maior percentual de área atingidas por maior profundidade de água.

Tabela 2. Proporção das profundidades das manchas de inundação com relação às

UDHs

\begin{tabular}{|c|c|c|c|c|c|c|c|c|c|c|}
\hline \multirow[t]{2}{*}{ UDH } & \multicolumn{2}{|c|}{ om } & \multicolumn{2}{|c|}{$0,1-1 \mathrm{~m}$} & \multicolumn{2}{|c|}{$1,1-2 m$} & \multicolumn{2}{|c|}{$2,1-3 m$} & \multicolumn{2}{|c|}{ Acima 3m } \\
\hline & $\begin{array}{l}\text { área } \\
\mathrm{km}^{2}\end{array}$ & $\begin{array}{c}\% \\
\text { UDH }\end{array}$ & $\begin{array}{l}\text { área } \\
\mathrm{km}^{2}\end{array}$ & $\%$ UDH & $\begin{array}{l}\text { área } \\
\mathrm{km}^{2}\end{array}$ & $\begin{array}{c}\% \\
\text { UDH }\end{array}$ & $\begin{array}{l}\text { área } \\
\mathrm{km}^{2}\end{array}$ & $\begin{array}{c}\% \\
\text { UDH } \\
\end{array}$ & $\begin{array}{l}\text { área } \\
\mathrm{km}^{2}\end{array}$ & $\begin{array}{c}\% \\
\text { UDH } \\
\end{array}$ \\
\hline Batel/Bigorrilho & 2,55 & 99,9 & 0,0 & 0,0 & 0,0 & 0,0 & 0,0 & 0,04 & 0,0 & 0,09 \\
\hline $\begin{array}{l}\text { Cabral/Hugo } \\
\text { Lange/Jd. } \\
\text { Social }\end{array}$ & 2,74 & 86,4 & 0,0 & 0,0 & 0,0 & 0,14 & 0,01 & 0,33 & 0,41 & 13,0 \\
\hline $\begin{array}{l}\text { Alto da } \\
\text { Glória/Juvevê/ } \\
\text { C. Cívico/Ahú }\end{array}$ & 2,05 & 89,5 & 0,0 & 0,0 & 0,0 & 0,0 & 0,0 & 0,13 & 0,24 & 10,3 \\
\hline $\begin{array}{l}\text { Alto da Rua } \\
\text { XV/Cristo Rei/ } \\
\text { Jd. Botânico }\end{array}$ & 4,66 & 84,2 & 0,0 & 0,0 & 0,0 & 0,0 & 0,0 & 0,0 & 0,87 & 15,7 \\
\hline $\begin{array}{l}\text { Jd. Botânico/ } \\
\text { Prado Velho: } \\
\text { Vila Torres }\end{array}$ & 0,83 & 87,1 & 0,02 & 1,53 & 0,04 & 4,49 & 0,03 & 2,78 & 0,04 & 3,86 \\
\hline $\begin{array}{c}\text { Centro/ } \\
\text { Rebouças }\end{array}$ & 0,91 & 67,1 & 0,0 & 0,01 & 0,01 & 0,60 & 0,03 & 1,96 & 0,41 & 30,1 \\
\hline Centro & 1,07 & 54,3 & 0,0 & 0,0 & 0,0 & 0,0 & 0,0 & 0,0 & 0,90 & 45,4 \\
\hline $\begin{array}{l}\text { Prado Velho/ } \\
\text { Rebouças }\end{array}$ & 1,69 & 99,4 & 0,0 & 0,0 & 0,01 & 0,56 & 0,0 & 0,02 & 0,0 & 0,0 \\
\hline Rebouças & 2,02 & 72,7 & 0,0 & 0,05 & 0,03 & 1,10 & 0,08 & 2,85 & 0,64 & 23,1 \\
\hline $\begin{array}{l}\text { Bom Retiro/ } \\
\text { Centro Cívico/ } \\
\text { Mercês/S. Fco. }\end{array}$ & 5,90 & 97,9 & 0,00 & 0,05 & 0,06 & 1,05 & 0,02 & 0,33 & 0,04 & 0,69 \\
\hline Água Verde & 2,159 & 99,9 & 0,0 & 0,0 & 0,0 & 0,05 & 0,0 & 0,0 & 0,0 & 0,0 \\
\hline
\end{tabular}

Fonte: autores, com base nos dados da pesquisa, 2019. 
Tabela 3 - Proporção de pessoas afetadas por UDH, considerando as profundidades atingidas pelas manchas de inundação

\begin{tabular}{|c|c|c|c|c|c|c|c|c|c|c|c|c|c|c|c|c|c|c|c|c|c|c|}
\hline \multirow{2}{*}{ UDH } & \multirow{2}{*}{\begin{tabular}{l|} 
Pop. \\
Total \\
UDH
\end{tabular}} & \multicolumn{4}{|c|}{ om } & \multicolumn{4}{|c|}{$0,1-1 \mathrm{~m}$} & \multicolumn{4}{|c|}{$1,1-2 m$} & \multicolumn{4}{|c|}{ 2,1-3m } & \multicolumn{4}{|c|}{ acima $3 \mathrm{~m}$} & \multirow{2}{*}{$\sum$ notas } \\
\hline & & peso & (a) & (b) & nota & peso & (a) & (b) & nota & peso & (a) & (b) & nota & peso & (a) & (b) & nota & peso & (a) & (b) & nota & \\
\hline $\begin{array}{c}\text { Batel/ } \\
\text { Bigorrilho }\end{array}$ & 29.728 & 0 & 29.712 & 99,9 & 0 & 0,25 & 0 & 0,0 & 0 & 0,5 & 0 & 0,0 & 0 & 0,75 & 5 & 0,02 & 0 & 1 & 11 & 0,04 & 0 & 0 \\
\hline $\begin{array}{l}\text { Cabral } \\
\text { H. Lange } \\
\text { Jd. Social }\end{array}$ & 27.536 & 0 & 26.204 & 95,2 & 0 & 0,25 & 0 & 0,0 & 0 & 0,5 & 20 & 0,07 & 0 & 0,75 & 36 & 0,13 & 0,1 & 1 & 1.275 & 4,6 & 4,6 & 4,7 \\
\hline $\begin{array}{l}\text { A. da Glória/ } \\
\text { Juvevê/ } \\
\text { C. Cívico/ } \\
\text { Ahú }\end{array}$ & 28.778 & 0 & 26.137 & 90,8 & 0 & 0,25 & 0 & 0,0 & 0 & 0,5 & 0 & 0,0 & 0 & 0,75 & 26 & 0,0 & 0 & 1 & 2.614 & 9,0 & 9,0 & 9,0 \\
\hline $\begin{array}{l}\text { A da XV } \\
\text { C. Rei/ Jd. } \\
\text { Botânico }\end{array}$ & 39.073 & 0 & 33.276 & 85,2 & 0 & 0,25 & 0 & 0,0 & 0 & 0,5 & 0 & 0,0 & 0 & 0,75 & 1 & 0,0 & 0 & 1 & 5.796 & 14,8 & 14,8 & 14,8 \\
\hline $\begin{array}{l}\text { Jd. Botânico/ } \\
\text { P. Velho: V. } \\
\text { Torres }\end{array}$ & 7.993 & 0 & 7.327 & 91,7 & 0 & 0,25 & 24 & 0,0 & 0 & 0,5 & 387 & 4,8 & 2,4 & 0,75 & 142 & 1,8 & 1,4 & 1 & 114 & 14,3 & 14,3 & 5,1 \\
\hline $\begin{array}{c}\text { Centro/ } \\
\text { Rebouças }\end{array}$ & 28.165 & 0 & 21.973 & 78,0 & 0 & 0,25 & 2 & 0,0 & 0 & 0,5 & 75 & 0,27 & 0,14 & 0,75 & 282 & 1,0 & 0,75 & 1 & 5.833 & 20,7 & 20,7 & 21,6 \\
\hline Centro & 26.858 & 0 & 18.737 & 69,8 & 0 & 0,25 & 0 & 0,0 & 0 & 0,5 & 0 & 0,0 & 0 & 0,75 & 3 & 0,01 & 0 & 1 & 8.118 & 30,2 & 30,2 & 30,2 \\
\hline $\begin{array}{l}\text { P. Velho/ } \\
\text { Rebouças }\end{array}$ & 9.949 & 0 & 9.900 & 99,5 & 0 & 0,25 & 0 & 0,0 & 0 & 0,5 & 47 & 0,47 & 0,23 & 0,75 & 2 & 0,02 & 0 & 1 & 0 & 0,0 & 0 & 0,23 \\
\hline Rebouças & 18.236 & 0 & 15.642 & 85,8 & 0 & 0,25 & 6 & 0,0 & 0 & 0,5 & 115 & 0,63 & 0,32 & 0,75 & 314 & 1,7 & 1,3 & 1 & 2.158 & 11,8 & 11,8 & 13,4 \\
\hline $\begin{array}{l}\text { B. Retiro/ } \\
\text { C. Cívico/ } \\
\text { Mercês/ } \\
\text { S. Fco. }\end{array}$ & 41.105 & 0 & 40.320 & 98,1 & 0 & 0,25 & 9 & 0,0 & 0 & 0,5 & 462 & 1,1 & 0,55 & 0,75 & 75 & 0,18 & 0,14 & 1 & 238 & 0,6 & 0,6 & 1,3 \\
\hline A. Verde & 32.153 & 0 & 32.146 & 99,9 & 0 & 0,25 & 0 & 0,0 & 0 & 0,5 & 7 & 0,0 & 0 & 0,75 & 0 & 0,0 & 0 & 1 & 0 & 0,0 & 0 & 0 \\
\hline
\end{tabular}
Fonte: autores, 2019. Com base nos dados da pesquisa.
(a) População projetada dentro da área na profundidade da mancha de inundação;
(b) Porcentagem da população afetada na UDH. 
A vulnerabilidade devido à inundação foi organizada em cinco classes de enquadramento. A partir das notas finais obtidas por cada UDH, fez-se a adoção das cinco classes e seus respectivos valores extremos para obter o intervalo entre as classes, fazendo uso da equação:

$\frac{\Delta \text { notas }}{N c}=$ I

Onde:

$\Delta$, é a variação entre a maior e a menor nota obtida pelas UDHs;

$\mathrm{Nc}$, é o número de classes de enquadramento de vulnerabilidade;

I, é o intervalo entre as classes de enquadramento de vulnerabilidade.

Considerando o número de classes como 5, a maior nota como sendo 30,2 (UDH: Centro) e o menor valor zero (UDH: Água Verde), obteve-se intervalo de classes de 6,04 para o índice de vulnerabilidade devido à inundações, demonstrado na Tabela 4.

Tabela 4. Classes de vulnerabilidade ambiental

\begin{tabular}{cc}
\hline Classes & Intervalo \\
\hline Muito baixa vulnerabilidade & zero a 6,04 \\
\hline Baixa vulnerabilidade & 6,04 a 12,08 \\
\hline Média vulnerabilidade & 12,08 a 18,12 \\
Alta vulnerabilidade & 18,12 a 24,16 \\
\hline Muito alta vulnerabilidade & 24,16 a 30,2 \\
\hline
\end{tabular}

Fonte: autor, 2019. Com base nos dados da pesquisa.

As UDH atingidas pelas manchas de inundação foram classificadas como: muito alta, alta, média, baixa e muito baixa vulnerabilidade, conforme apresentadas na Tabela 5.

Tabela 5. Índices de vulnerabilidade das UDHs atingidas por manchas de inundação

\begin{tabular}{lcc}
\hline \multicolumn{1}{c}{ UDH } & IV & Classe de vulnerabilidade \\
\hline Batel/Bigorrilho & 0,0 & Muito baixa vulnerabilidade \\
\hline Cabral/Hugo Lange/Jd. Social & 4,7 & Muito baixa vulnerabilidade \\
\hline Alto da Glória/Juvevê/ C. Cívico/Ahú & 9,0 & Baixa vulnerabilidade \\
\hline Alto da Rua XV/Cristo Rei/ Jd. Botânico & 14,8 & Média vulnerabilidade \\
\hline Jd. Botânico/Prado Velho: Vila Torres & 5,1 & Muito baixa vulnerabilidade \\
\hline Centro/Rebouças & 21,6 & Alta vulnerabilidade \\
\hline Centro & 30,2 & Muito alta vulnerabilidade \\
\hline Prado Velho/Rebouças & 0,23 & Muito baixa vulnerabilidade \\
\hline Rebouças & 13,4 & Média vulnerabilidade \\
\hline Bom Retiro/Centro Cívico/ Mercês/S. Fco. & 1,3 & Muito baixa vulnerabilidade \\
\hline Água Verde & 0,0 & Muito baixa vulnerabilidade \\
\hline
\end{tabular}

Fonte: autores, 2019. Com base nos dados da pesquisa. 
Figura 2. UDH atingidas pelas manchas de inundação simuladas, com valores de IVA

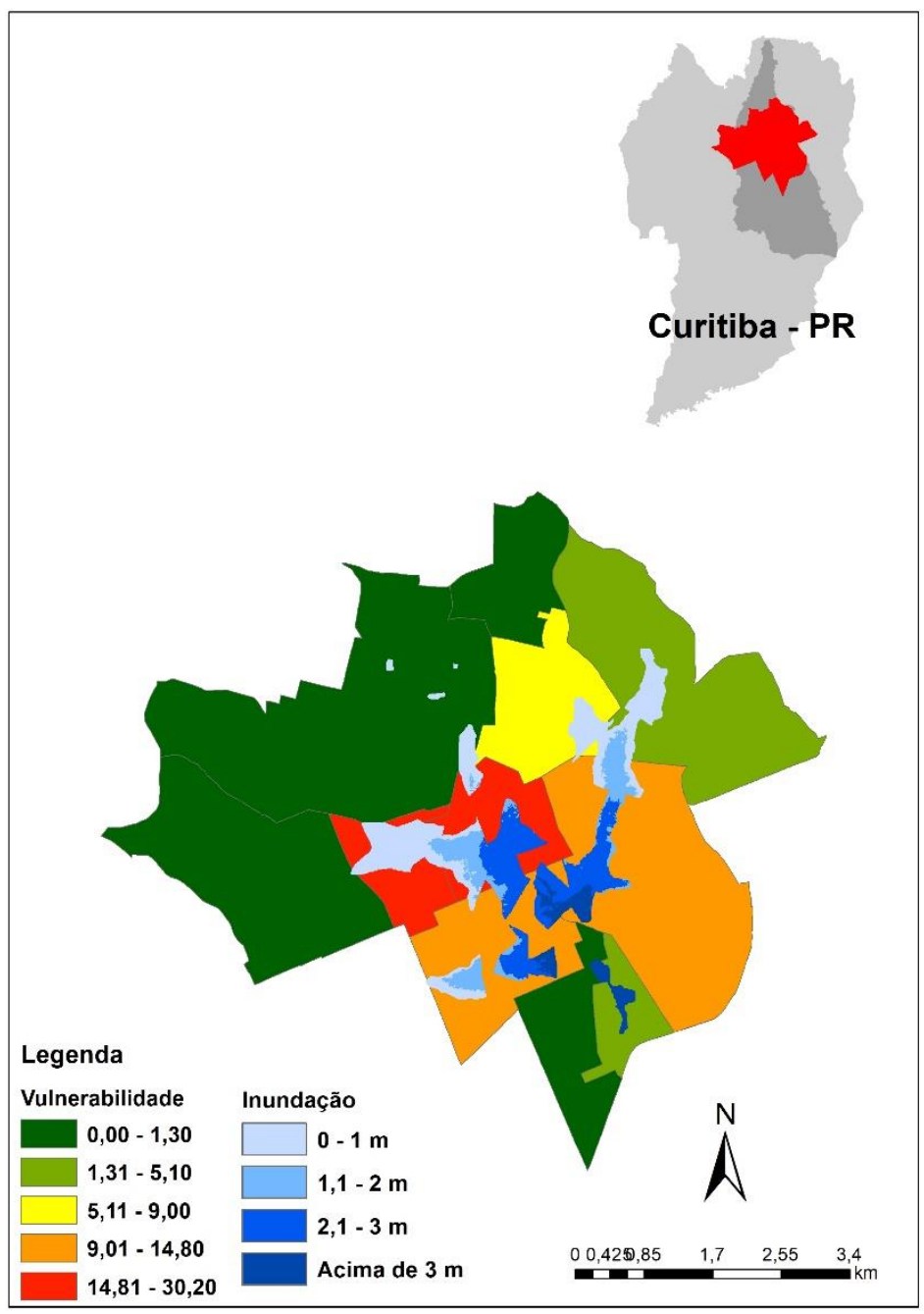

Fonte: autores, 2019. Com base nos dados da pesquisa.

A vulnerabilidade social de uma UDH pode variar entre 5 classes, sendo: muito baixa vulnerabilidade; baixa vulnerabilidade; média vulnerabilidade; alta vulnerabilidade; e muito alta vulnerabilidade. Para possibilitar a visualização da vulnerabilidade social da área de estudo, os dados de vulnerabilidade social foram processados e espacializados em ambiente SIG, gerando mapas temáticos do IVS da área de estudo.

A Tabela 6 apresenta o IVS normalizado das UDH atingidas pelas manchas de inundação e a vulnerabilidade indicada pelas mesmas. Essa normalização se fez necessária para que se pudesse associar o IVS à vulnerabilidade devido às inundações. Assim, os dois índices estarão dentro da mesma escala e no mesmo número de classes de vulnerabilidade.

Nota-se que grande parte das UDHs atingidas pelas potenciais manchas de inundação se enquadram na classe de vulnerabilidade social muito baixa, e contam com maior contagem populacional, bem como contam com maiores rendas domiciliares médias.

Apenas a UDH Jardim Botânico/Prado Velho: Vila das Torres se enquadra na categoria muito alta vulnerabilidade, sendo que os valores das três dimensões do IVS: 
Infraestrutura Urbana, Capital Humano e Renda e Trabalho para esta UDH se destacam contundentemente dos demais, demonstrando grande discrepância entre esta população e esta UDH frente às demais analisadas.

De forma que os três indicadores visam refletir a habitabilidade das pessoas, seu estoque de capital humano suas condições de renda, apresentam-se nesta UDH a parcela de população com maiores fragilidades e com menor resiliência frente à eventos de desastres naturais, tendo maior propensão de sofrerem maiores perdas e danos, bem como podem demonstrar maior tempo de recuperação.

Tabela 6. UDH da bacia hidrográfica do Rio Belém afetadas por manchas de inundação

\begin{tabular}{|c|c|c|c|c|}
\hline Nome UDH & Classe IVS & $\begin{array}{c}\text { IVS } \\
\text { normalizado }\end{array}$ & Pop. UDH & $\begin{array}{c}\text { Renda média } \\
\text { UDH }\end{array}$ \\
\hline Batel / Bigorrilho & Muito baixa & 4,2 & 29.728 & $\mathrm{R} \$ 2.837,25$ \\
\hline Cabral / Hugo Lange/ Jardim Social & Muito baixa & 4,2 & 27.536 & $\mathrm{R} \$ 2.694,67$ \\
\hline $\begin{array}{l}\text { Alto da Glória / Juvevê / Centro } \\
\text { Cívico / Ahú }\end{array}$ & Muito baixa & 4,9 & 28.778 & $R \$ 3.061,56$ \\
\hline $\begin{array}{l}\text { Alto da Rua XV / Cristo Rei / Jardim } \\
\text { Botânico }\end{array}$ & Muito baixa & 4,9 & 39.073 & $R \$ 2.465,26$ \\
\hline $\begin{array}{l}\text { Jardim Botânico / Prado Velho: Vila } \\
\text { das Torres }\end{array}$ & Muito alta & 33,4 & 7.993 & $\mathrm{R} \$ 1.477,77$ \\
\hline Centro / Rebouças & Muito baixa & 6,5 & 28.165 & $R \$ 3.025,55$ \\
\hline Centro & Muito baixa & 4,6 & 26.858 & $R \$ 2.515,16$ \\
\hline Prado Velho / Rebouças & Média & 18,6 & 9.949 & $\mathrm{R} \$ 1.534,95$ \\
\hline Rebouças & Baixa & 8 & 18.236 & $\mathrm{R} \$ 1.880,33$ \\
\hline $\begin{array}{l}\text { Bom Retiro / Centro Cívico / Mercês } \\
\text { / S. Francisco }\end{array}$ & Média & 18,7 & 41.105 & $\mathrm{R} \$ 2.487,43$ \\
\hline Água Verde & Muito baixa & 5,2 & 32.153 & $\mathrm{R} \$ 2.542,58$ \\
\hline
\end{tabular}

Fonte: autores, 2019. Com base nos dados da pesquisa. 
Figura 3. UDH atingidas pelas manchas de inundação, com valores do IVS

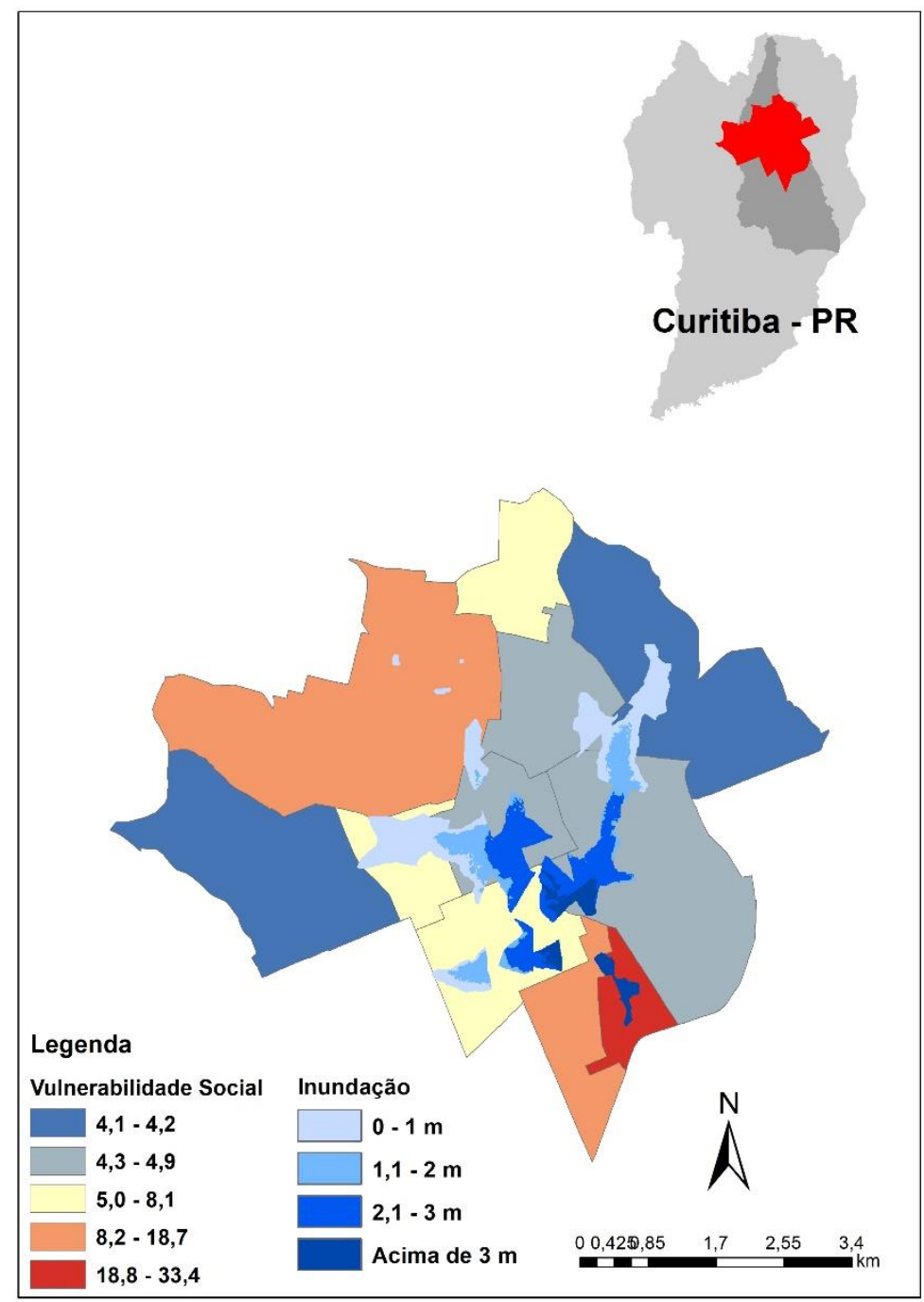

Fonte: autores, 2019. Com base nos dados da pesquisa.

Enquanto o IVS apresenta enfoque no "acesso, ausência ou insuficiência de ativos que deveriam estar à disposição da população [...]" (IPEA, 2015, p.12) nas suas três grandes dimensões: Infraestrutura urbana - acesso à serviços de saneamento básico e mobilidade urbana; Capital Humano - acesso à serviços de saúde e educação e; Renda e Trabalho - insuficiência de renda com base no salário mínimo do ano de 2010, ocupação da população, dependência de renda de idosos e trabalho infantil (IPEA, 2015).

O índice de vulnerabilidade de inundações sinaliza possível propensão a esse tipo de evento em áreas do território urbano, e relaciona as profundidades atingidas pela inundação simulada à possíveis populações residentes na área impactada pelas manchas. Ambos os índices contam com cinco classes de vulnerabilidade (Tabela 7), que demonstram o enquadramento dos índices nas categorias: muito baixa vulnerabilidade, baixa vulnerabilidade, média vulnerabilidade, alta vulnerabilidade e muito alta vulnerabilidade.

$\mathrm{Na}$ associação entre os índices de vulnerabilidades social e vulnerabilidade devido às inundações resultou em um índice geral de vulnerabilidade, aqui 
denominado de IVSI (Índice de vulnerabilidade socioambiental devido às inundações), apresentado na Tabela 7.

Tabela 7. Índices de vulnerabilidade social e ambiental para as UDHs

\begin{tabular}{|c|c|c|c|c|c|}
\hline Nome da UDH & $\begin{array}{c}\text { Classe de } \\
\text { vulnerabilidade } \\
\text { social }\end{array}$ & $\begin{array}{l}\text { Índice } \\
\text { IVS }\end{array}$ & $\begin{array}{c}\text { Classe de } \\
\text { vulnerabilidade } \\
\text { ambiental } \\
\end{array}$ & Índice IV & $\Sigma$ \\
\hline Batel/Bigorrilho & Muito baixa & 4,2 & Muito baixa & 0 & 4,2 \\
\hline $\begin{array}{l}\text { Cabral/Hugo Lange/Jd. } \\
\text { Social }\end{array}$ & Muito baixa & 4,2 & Muito baixa & 4,7 & 8,9 \\
\hline $\begin{array}{l}\text { Alto da Glória/Juvevê/ } \\
\text { Centro Cívico/Ahú }\end{array}$ & Muito baixa & 4,9 & Baixa & 9 & 13,9 \\
\hline $\begin{array}{l}\text { Alto da Rua XV/Cristo } \\
\text { Rei/ Jardim Botânico }\end{array}$ & Muito baixa & 4,9 & Média & 14,8 & 19,7 \\
\hline $\begin{array}{l}\text { Jardim Botânico/Prado } \\
\text { Velho: Vila das Torres }\end{array}$ & Muito alta & 33,4 & Muito baixa & 5,1 & 38,5 \\
\hline Centro/Rebouças & Muito baixa & 6,5 & Alta & 21,6 & 28,1 \\
\hline Centro & Muito baixa & 4,6 & Muito alta & 30,2 & 34,8 \\
\hline Prado Velho/Rebouças & Média & 18,6 & Muito baixa & 0,23 & 18,83 \\
\hline Rebouças & Baixa & 8 & Média & 13,4 & 21,4 \\
\hline $\begin{array}{l}\text { Bom Retiro/Centro } \\
\text { Cívico/ Mercês/São } \\
\text { Francisco }\end{array}$ & Média & 18,7 & Muito baixa & 1,3 & 20 \\
\hline Água Verde & Muito baixa & 5,2 & Muito baixa & 0 & 5,2 \\
\hline
\end{tabular}

Fonte: autores, 2019. Com base nos dados da pesquisa.

O processo de classificação de do Índice de Vulnerabilidade Socioambiental devido às inundações (IVSI) seguiu o mesmo procedimento utilizado na conformação do índice de vulnerabilidade devido às inundações, onde considerou-se o número de classes como 5, o maior valor de nota entre as UDHs como sendo 38,5 (UDH: Jardim Botânico/Prado Velho: Vila das Torres) e o menor valor sendo 4,2 (UDH: Batel/Bigorrilho), com o intervalo para classificação de vulnerabilidade socioambiental devido às inundações obtido sendo 6,86 , que pode ser observado na Tabela 8.

Tabela 8. Classes de vulnerabilidade socioambiental

\begin{tabular}{cc}
\hline Classes & Intervalo \\
\hline Muito baixa vulnerabilidade & 4,2 a 11,06 \\
\hline Baixa vulnerabilidade & 11,06 a 17,92 \\
\hline Média vulnerabilidade & 17,92 a 24,78 \\
\hline Alta vulnerabilidade & 24,78 a 31,64 \\
\hline Muito alta vulnerabilidade & 31,64 a 38,5
\end{tabular}

Fonte: autores, 2019. Com base nos dados da pesquisa. 
O IVSI é o resultado do somatório dos índices IVS e IV, sendo que cada um deles se insere no cálculo com o mesmo peso. As UDH atingidas pelas manchas de inundação foram classificadas nas classes: muito alta, alta, média, baixa e muito baixa vulnerabilidade ambiental, conformando o índice IVSI - Índice de Vulnerabilidade Socioambiental devido às inundações, como demonstrado na Erro! Fonte de referência não encontrada.9, enquanto a Erro! Fonte de referência não encontrada. apresenta as informações de forma espacial.

Observa-se que a vulnerabilidade ambiental contribuiu em grande parte para a reclassificação das UDH no índice IVSI, especialmente na região central analisada (UDHs: Centro, Rebouças e Centro/Rebouças), que concentra grande impacto não apenas na extensão territorial das manchas de inundação, mas também nas possíveis profundidades atingidas pelas mesmas.

Tabela 9. Índice IVSI para as UDHs

\begin{tabular}{ccc}
\hline Nome da UDH & $\begin{array}{c}\text { Classe de } \\
\text { vulnerabilidade } \\
\text { socioambiental }\end{array}$ & Índice IVSI \\
\hline Batel/Bigorrilho & Muito baixa & 4,2 \\
\hline Cabral/Hugo Lange/Jd. Social & Muito baixa & 8,9 \\
\hline Alto da Glória/Juvevê/ Centro Cívico/Ahú & Baixa & 13,9 \\
\hline Alto da Rua XV/Cristo Rei/ Jardim Botânico & Média & 19,7 \\
\hline Jardim Botânico/Prado Velho: Vila das & Muito alta & 38,5 \\
Torres & Alta & 28,1 \\
\hline Centro/Rebouças & Alta & 34,8 \\
\hline Centro & Média & 18,83 \\
\hline Prado Velho/Rebouças & Média & 21,4 \\
\hline Rebouças & Média & 20 \\
\hline Brancisco & Muito baixa & 5,2 \\
\hline
\end{tabular}

Fonte: autores, 2019. Com base nos dados da pesquisa. 
Figura 4.Índice IVSI para as UDHs do recorte geográfico

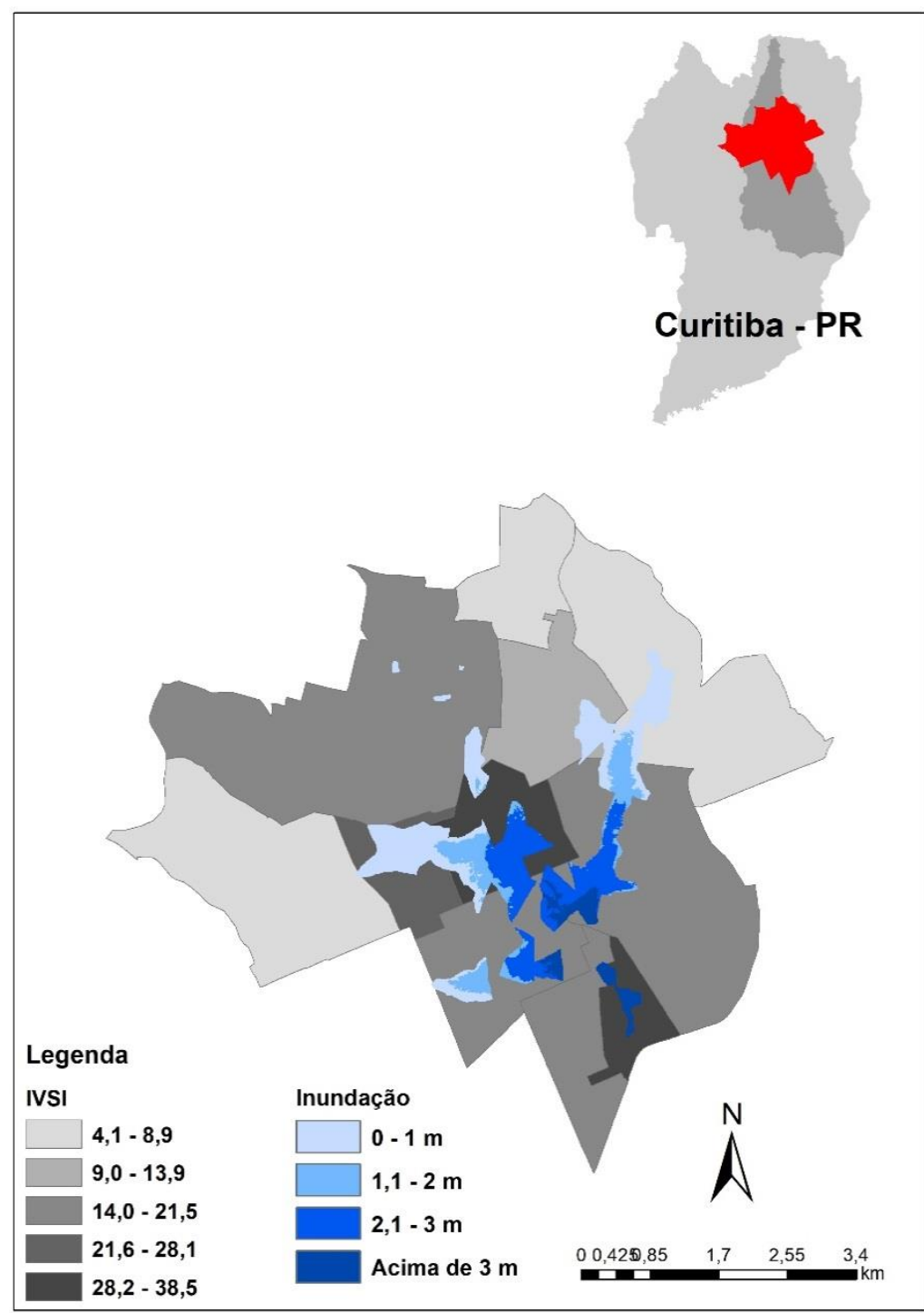

Fonte: autores, 2019. Com base nos dados da pesquisa.

Dentre as UDH impactadas pelas manchas de inundação simuladas, destacouse a UDH denominada Jardim Botânico/Prado Velho: Vila das Torres, que possui maior índice de vulnerabilidade social (IVS 33,4), menor média de renda entre as 11 UDHs da área de estudo ( $\mathrm{R} \$ 1.477,77)$, menor população (7.993 pessoas), e classificou-se como muito baixa vulnerabilidade ambiental devido às inundações (IV 5,1 ) considerando o impacto das manchas de inundação. Devido ao fato de esta porção populacional ter aspectos característicos, e a articulação das vulnerabilidades social e ambiental devido às inundações demonstrou incremento significativo no índice IVSI $(38,5)$ no contexto das UDHs analisadas, procurou-se contextualizar a UDH Vila das Torres frente às demais UDHs impactadas.

A primeira intervenção do poder público no local da Vila das Torres data de 1976, com a promoção do desfavelamento da ocupação, com justificativa de implantação dos equipamentos Jardim Botânico e campus da Universidade Federal do Paraná nas proximidades (SOUZA, 2016). Esta política pública de erradicação de favelas fez parte da atuação do Governo Federal, por meio do Banco Nacional de Habitação (BNH), durante a ditatura militar (BUENO, 2000). 
A fixação do assentamento Vila das Torres data do final da década de 1980, com execução de infraestrutura mínima e regularização pelo poder público, com recursos provenientes do Governo Federal. Esta intervenção toma partido das políticas públicas de urbanização, onde mantêm-se as características do local, com implantação de alterações que promovam melhoria da infraestrutura, objetivando maior qualidade de vida aos moradores (BUENO, 2000; DENALDI, 2003).

Com o estabelecimento e regularização da Vila Torres, diversas outras intervenções de complementação urbanística da área foram implementadas ao longo do tempo, com fornecimento de serviços básicos de infraestrutura de saneamento, consolidação do sistema viário e alterações e melhorias nas edificações, como o Programa Comunidade em Cores, lançado pela COHAB-CT em 2010 (SOUZA, 2016).

Verifica-se no processo de urbanização do local que apenas a primeira política pública aplicada pela Prefeitura de Curitiba teve intuito de remoção da população, todas as políticas posteriores são caracterizadas como urbanização. Embora o local seja atualmente considerado regularizado pelo poder público, a questão fundiária é tema sensível, apresentando quadro de urbanização precário, tendo a área características de não-integração com a cidade formal (BUENO, 2000; DENALDI, 2003; SOUZA, 2016).

A consolidação da ocupação nas margens do Rio Belém pelo poder público vai de encontro com a legislação vigente, que determina a não ocupação ou desenvolvimento de atividades com impacto ambiental em margens de rios, córregos, nascentes e demais estruturas hidrológicas. Esta consideração enfatiza a consolidação do risco no ambiente urbano, reduzindo a resiliência da estruturação urbana e incrementando a vulnerabilidade social, além de salientar problemas de ordem jurídica, pois diferentes esferas legislativas têm entendimentos divergentes na aplicação das leis em vigência.

Mesmo localizada na região central da cidade de Curitiba, a UDH onde se encontra a Vila Torres demonstra características muito particulares. De forma que a ocupação da região da Vila das Torres se deu por estabelecimento de população vulnerável em local de risco, diversas são as implicações da anuência de regularização dessa população pelo poder público, ocasionando maximização dos indicadores de vulnerabilidade social, bem como sedimenta a dinâmica de segregação urbana, visto que a inserção do local na "cidade formal" se deu de forma lenta, com ações pontuais de saneamento e infraestrutura pela Prefeitura Municipal de Curitiba e entidades ligadas à mesma.

Notou-se também que grande área das manchas de inundação simuladas na porção central da bacia hidrográfica de estudo toma forma em UDHs com menores índices de vulnerabilidade social dentre as analisadas (UDHs Centro e Centro/Rebouças). Retomando os estudos de Rolnik (1988) e Villaça (2001), traçouse um paralelo entre as diferentes produções de localizações nos centros urbanos e esta mesma produção aplicada à área central da bacia hidrográfica do Rio Belém.

Considerando os indicadores de vulnerabilidade do IPEA, baixa vulnerabilidade social compreende acesso e usufruto de serviços de saneamento urbano (abastecimento, esgotamento e coleta de resíduos sólidos, além do controle de vetores), acesso ao sistema de ensino, ocupação da população em atividades remuneradas formais, menor número de núcleos familiares monoparentais, entre outras atribuições para designação de vulnerabilidade social. Diante destes aspectos, 
identificou-se na conformação do ambiente urbano da área de estudo proposta, que a mesma seguiu o processo de urbanização excludente apontado por Maricato (2000) e Rolnik e Klink (2011), onde populações com maiores faixas de renda tendem a se localizar em regiões das cidades com maior oferta de infraestrutura urbana e social.

Ainda que estas características de inclusão e acesso conformem diminuta vulnerabilidade social, não pode-se dizer o mesmo com relação à vulnerabilidade ambiental aos processos de inundações simulados, pois grande parte da porção central da Bacia Hidrográfica do Rio Belém demonstrou propensão a estes eventos, indicando não apenas problemas graves de possíveis inundações urbanas, mas problemas na infraestrutura de drenagem urbana, em grande parte ocasionadas pela massiva impermeabilização do solo, bem como maior pressão no sistema de saneamento pela crescente densidade demográfica.

\section{Conclusão}

Os princípios do ciclo hidrológico - evaporação, precipitação, infiltração e escoamento superficial - para manterem-se em equilíbrio, necessitam de compensações caso haja alterações nas estruturas básicas do sistema. Quando o sistema hidrológico não atinge um nível satisfatório de equilíbrio, desenvolvem-se os desastres naturais, se os mesmos ocorrerem em aglomerados urbanos.

O sistema antrópico, com sua forma complexa e interdisciplinar, desenvolveuse a partir de parâmetros excludentes e seletivos, que podem ser observados, confirmados e vivenciados, apresentando-se de forma contundente nos países das Américas Latina e Central, África e Ásia. No caso específico do Brasil, diversas são as pesquisas e estudos que demonstram o abismo - tanto no âmbito social, quanto no âmbito ambiental, das condições da sociedade e do uso do território.

Neste sentido, a congregação de indicadores sociais e ambientais desenvolvido nesta pesquisa motiva o estreitamento da relação natureza-sociedade, onde os processos sociais, em sua maioria, determinam o acesso à oportunidades e desastres, e podem possuir distribuição desigual, considerando os sistemas sociais e relações de poder existentes, que podem conformar panoramas de vulnerabilidade, em casos onde o não-acesso à recursos evidencia-se, mas também pode revelar aspectos de resiliência, com readaptação eficiente da população à novas realidades.

Na questão da espacialização de cenários de inundação simulados, destacouse a utilização do método Hazus, que inseriu a variável "profundidade" nas análises da vulnerabilidade ambiental e posterior vulnerabilidade socioambiental devido às inundações, caracterizando a população atingida em níveis de profundidade da massa hídrica, cruzando a caracterização ambiental com as características de enquadramento da população residente no IVS. Esta relação entre a localização da população, suas características, e possível cenário de inundação urbana podem fornecer subsídio para a gestão pública e sociedade, visando a redução de impactos oriundos de desastres naturais, bem como direcionamentos no que diz respeito à regulamentação da utilização de determinadas áreas das cidades, e obras de melhorias e/ou readequações na infraestrutura urbana.

De forma que a metodologia Hazus encontra-se em estágio inicial de aplicação no Brasil, a mesma vem sendo aprimorada no que diz respeito à inserção de dados 
no sistema, que originalmente segue a convenção dos modelos norte-americanos, com diferentes técnicas e materiais de construção, bem como diferentes unidades de medida. Visto que a aplicação da presente metodologia Hazus demanda substancial trabalho na etapa de aquisição e tratamento de dados, necessita-se de recursos humanos altamente capacitados para que sejam realizadas as alterações necessárias na programação do software, bem como para posterior análise dos dados obtidos.

Ainda que haja grande afastamento entre a realidade americana e brasileira no que diz respeito à aplicação da metodologia Hazus, a mesma é possível e segue acompanhada de oportunidades no âmbito da modelagem computacional voltada para prevenção e recuperação de desastres naturais, no auxílio da estruturação de uma sociedade resiliente e preparada para eventos de desastres naturais.

\section{Agradecimentos}

Os autores gostariam de agradecer ao Conselho Nacional de Desenvolvimento Científico e Tecnológico (CNPq), ao Centro Universitário de Estudos e Pesquisas sobre Desastres do Paraná (CEPED/PR) e à SANEPAR pelo apoio financeiro ao projeto de pesquisa. E a Federal Emergency Management Agency (FEMA) pelo suporte técnico para adaptação do Hazus para o Brasil.

\section{REFERÊNCIAS}

BOLLMANN, H. A.; EDWIGES, T. Avaliação da qualidade das águas do Rio Belém, Curitiba-PR, com emprego de indicadores quantitativos e perceptivos. Engenharia Sanitária Ambiental, Rio de Janeiro, vol.13, n.4, p. 443-452, out/dez. 2008.

DING, A.; WHITE, J. F.; ULLMAN, P. W.; FASHOKUN, A. O. Evaluation of HAZUS-MH flood model with local data and other program. Natural Hazards Review, Reston, v.9, n.1, p.20-28, fev. 2008.

HUMMEL, B. L.; CUTTER, S. L.; EMRICH, C. T. Social vulnerability to natural hazards in Brazil. International Journal of Disaster Risk Science, Pequim, v.7, n.2, p.111-122, jun. 2016. INSTITUTO BRASILEIRO DE GEOGRAFIA E ESTATÍSTICA - IBGE. Censo demográfico 2010. Rio de Janeiro: IBGE, 2010.

INSTITUTO PARANAENSE DE DESENVOLVIMENTO ECONÔMICO E SOCIAL - IPARDES. Índice de vulnerabilidade das famílias paranaenses: mensuração a partir do Cadastro Único para Programas Sociais - Cadúnico. Nota técnica IPARDES, Curitiba, dez. 2012.

INSTITUTO DE PESQUISA ECONÔMICA APLICADA - IPEA. Atlas da vulnerabilidade social nos municípios brasileiros. Brasília: IPEA, 2015.

JONOV, C. M. P.; NASCIMENTO, N. de O.; PAULA E SILVA, A. de. Avaliação de danos às edificações causados por inundações e obtenção dos custos de recuperação. Ambiente Construído, Porto Alegre, v.13, n.3, p.75-94, jan./mar. 2013.

MACHADO, M. L. et al. Curvas de danos de inundações versus profundidade de submersão: desenvolvimento de metodologia. Revista de Gestão de Água da América Latina - REGA, Porto Alegre, v.02, n.01, p.35-52, jan/jun. 2005. 
MARICATO, E. Metrópole, legislação e desigualdade. Estudos avançados, São Paulo, v.17, n.48, p.151-167, mai/ago. 2003.

ROLNIK, R.; KLINK, J. Crescimento econômico e desenvolvimento urbano: Por que nossas cidades continuam tão precárias?. Novos Estudos, São Paulo, v.30, n.01, p.89-109, mar. 2011.

SCHNEIDER, P.J.; SCHAUER, B. A. HAZUS - its development and its future. Natural Hazards Review, Reston, n.2, v.7, mai. 2006.

SCAWTHORN, C. et al. Hazus-MH flood loss estimation methodology: overview and flood hazard characterization. Natural Hazards Review, Reston, n.2, v.7, mai. 2006.

SUPERINTENDÊNCIA DE DESENVOLVIMENTO DE RECURSOS HÍDRICOS E SANEAMENTO AMBIENTAL - SUDERHSA. Plano Diretor de Drenagem para a Bacia do Rio Iguaçu na Região Metropolitana de Curitiba. Curitiba: SUDERHSA, 2002.

TINGSANCHALI, T. Urban flood disaster management. Procedia Engeneering, Nova York, v.32, p.25-37, nov. 2012.

TUCCI, C. E. M. Águas urbanas. Estudos avançados, São Paulo, v.22, n.63, p.97-112, jun. 2008. VEIT, E. A; ARAÚJO, I. S. Modelagem computacional aplicada ao ensino de ciências. In: MOREIRA, M. A; VEIT, E. A. (Org.) Ensino superior: bases teóricas e metodológicas. São Paulo: E.P.U, 2010. 224p.

Edilberto Nunes Moura. Professor titular da Pontifícia Universidade Católica do Paraná - PUCPR, no Programa de Pós-Graduação em Gestão Urbana - PPGTU (Mestrado/Doutorado).edilberto.moura@pucpr.br

Ariadne Stradiotto Frenzel. Mestrado em andamento em Gestão Urbana. ariadne.frenzel@gmail.com

Isabela Ignácio de Moura. Estudante de Arquitetura e Urbanismo, Universidade Tecnológica Federal do Paraná (UTFPR). Atualmente é estagiária em Arquitetura e Urbanismo. isabela.igmoura@gmail.com em: https://doi.org/10.17058/redes.v24i2.13251. 